\title{
Exponential collocation methods for conservative or dissipative systems
}

\author{
Bin Wang * $\quad$ Xinyuan $\mathrm{Wu}^{\dagger}$
}

September 18, 2018

\begin{abstract}
In this paper, we propose and analyse a novel class of exponential collocation methods for solving conservative or dissipative systems based on exponential integrators and collocation methods. It is shown that these novel methods can be of arbitrarily high order and exactly or nearly preserve first integrals or Lyapunov functions. We also consider order estimates of the new methods. Furthermore, we explore and discuss the application of our methods in important stiff gradient systems, and it turns out that our methods are unconditionally energydiminishing and strongly damped even for very stiff gradient systems. Practical examples of the new methods are derived and the efficiency and superiority are confirmed and demonstrated by three numerical experiments including a nonlinear Schrödinger equation. As a byproduct of this paper, arbitrary-order trigonometric/RKN collocation methods are also presented and analysed for second-order highly oscillatory/general systems. The paper is accompanied by numerical results that demonstrate the great potential of this work. Keywords: exponential integrators, energy-preserving algorithms, first integral, Lyapunov function, finite element methods, collocation methods
\end{abstract}

MSC: 65L05, 65L60, 65P10

\section{Introduction}

In this paper, we are concerned with systems of ordinary differential equations (ODEs) of the form

$$
y^{\prime}(t)=Q \nabla H(y(t)), \quad y(0)=y_{0} \in \mathbb{R}^{d}, \quad t \in[0, T],
$$

where $Q$ is an invertible and $d \times d$ real matrix, and $H: \mathbb{R}^{d} \rightarrow \mathbb{R}$ is defined by

$$
H(y)=\frac{1}{2} y^{\top} M y+V(y) .
$$

*School of Mathematical Sciences, Qufu Normal University, Qufu 273165, P.R. China; Mathematisches Institut, University of Tübingen, Auf der Morgenstelle 10, 72076 Tübingen, Germany. The research is supported in part by the Alexander von Humboldt Foundation and by the Natural Science Foundation of Shandong Province (Outstanding Youth Foundation) under Grant ZR2017JL003. E-mail: wang@na.uni-tuebingen.de

${ }^{\dagger}$ School of Mathematical Sciences, Qufu Normal University, Qufu 273165, P.R. China; Department of Mathematics, Nanjing University, Nanjing 210093, P.R. China. The research is supported in part by the National Natural Science Foundation of China under Grant 11671200. E-mail: xywu@nju.edu.cn 
Here $M$ is a $d \times d$ symmetric real matrix, and $V: \mathbb{R}^{d} \rightarrow \mathbb{R}$ is a differentiable function.

It is important to note that the system (11) exhibits remarkable geometrical/physical structures. If the matrix $Q$ is skew symmetric, then (10) is a conservative system with the first integral $H$ : i.e.,

$$
H(y(t)) \equiv H\left(y_{0}\right) \quad \text { for any } t \geq 0 .
$$

When the matrix $Q$ is negative semidefinite, then (11) is a dissipative system with the Lyapunov function $H$ : i.e.,

$$
H\left(y\left(t_{2}\right)\right) \leq H\left(y\left(t_{1}\right)\right) \text { if } t_{2} \geq t_{1} .
$$

In this paper, we call $H$ energy for both cases in a broad sense. The objective of this paper is to design and analyse a class of novel arbitrary-order exponential energy-preserving collocation methods to preserve first integrals or Lyapunov functions of the conservative/dissipative system (11).

For brevity, let

$$
A=Q M, \quad g(y(t))=Q \nabla V(y(t)) .
$$

We then rewrite the system (10) as

$$
y^{\prime}(t)=A y(t)+g(y(t)), \quad y(0)=y_{0} \in \mathbb{R}^{d} .
$$

As is known, the exact solution of (11) or (3) can be represented by the variation-of-constants formula (the Duhamel Principle)

$$
y(t)=e^{t A} y_{0}+t \int_{0}^{1} e^{(1-\tau) t A} g(y(\tau t)) d \tau .
$$

The system (11) or (3) plays a prominent role in a wide range of applications in physics and engineering, inclusive of mechanics, astronomy, molecular dynamics, and in problems of wave propagation in classical and quantum physics (see, e.g. [25, 33, 58, 62]). Some highly oscillatory problems and semidiscrete PDEs such as semilinear Schrödinger equations all fit this form. One important example of them is the multi-frequency highly oscillatory Hamiltonian systems with the following Hamiltonian

$$
H(q, p)=\frac{1}{2} p^{\top} \bar{M}^{-1} p+\frac{1}{2} q^{\top} \bar{K} q+U(q),
$$

where $\bar{K}$ is a symmetric positive semi-definite stiffness matrix, $\bar{M}$ is a symmetric positive definite mass matrix, and $U(q)$ is a smooth potential with moderately bounded derivatives.

In recent decades, exponential integrators have been widely investigated and developed as an efficient approach to integrating (3), and we refer the reader to [3, 8, 9, 11, 12, 13, 19, 22, 31, 34, 47, 56, 60] for example. Exponential integrators make well use of the variation-of-constants formula (4), and their performance has been evaluated by a range of test problems. A systematic survey of exponential integrators is referred to 33. However, apart from symplectic exponential integrators (see, e.g. 44]), most existing publications dealing with exponential integrators focus on the construction and analysis of the schemes and never consider deriving energy-preserving exponential integrators to preserve the first integrals/Lyapunov functions. Energy-preserving exponential integrators, especially higher-order schemes of them have not been well researched yet in the literature.

On the other hand, various effective energy-preserving methods have been proposed and researched for (3) in the special case of $A=0$, such as the average vector field (AVF) method 14, 15, 48, discrete gradient (DG) methods [41, 43, 52, Hamiltonian Boundary Value Methods 
(HBVMs) 6, 7, the Runge-Kutta-type energy-preserving collocation (RKEPC) methods [17, 23, time finite elements (TFE) methods 4, 5, 27, 38, 49, and energy-preserving exponentially-fitted (EPEF) methods 45, 46. Some numerical methods preserving Lyapunov functions have also been studied for (3) with $A=0$ (see, e.g. [10, 24, 42). It is noted that all these methods are constructed and studied for the special case $A=0$ and thus they do not take advantage of the structure brought by the linear term $A y$ in the system (3). These methods could be applied to (3) with $A \neq 0$ if the right-hand side of (3) is considered as a whole function: i.e., $y^{\prime}=f(y) \equiv A y-g(y)$.

Recently, in order to take advantage the structure of the underlying system and preserve its energy simultaneously, a novel energy-preserving method has been studied in [54, 59, for secondorder ODEs and a new energy-preserving exponential scheme for the conservative or dissipative system has been researched in 37. However, these two kinds of methods are both based on the AVF methods and thence they are only of order two, in general. This results in insufficiency to deal with some practical problems for high-precision numerical simulations in sciences and engineering.

On the basis of the facts stated above, this paper is devoted to deriving and analysing novel exponential collocation methods. For this purpose, we make well use of the variation-of-constants formula and the structure introduced by the underlying system. In such a way, these exponential integrators can exactly or nearly preserve the first integral or the Lyapunov function of (1D).

The paper is organised as follows. We first formulate the exponential collocation methods for first-order ODEs (10) in the next section. As a byproduct, the trigonometric/RKN collocation methods for second-order systems are presented in Section 3. Section 4 pays attention to showing that the novel methods preserve exactly or nearly first integrals or Lyapunov functions. From Section 5 to Section 6 , the properties of the methods are discussed in detail including existence and uniqueness, and algebraic order. In Section 7, we discuss the application of our methods to stiff gradient systems. Illustrative examples of the new methods are derived in Section 8 and numerical experiments are presented in Section 9. Finally, this paper ends with some concluding remarks and discussions.

\section{Formulation of new methods}

Following [38, we define the finite-dimensional function spaces $Y_{h}$ as follows:

$$
\begin{aligned}
Y_{h} & =\operatorname{span}\left\{\tilde{\varphi}_{0}(\tau), \ldots, \tilde{\varphi}_{r-1}(\tau)\right\} \\
& =\left\{\tilde{w}: \tilde{w}(\tau)=\sum_{i=0}^{r-1} \tilde{\varphi}_{i}(\tau) W_{i}, \tau \in[0,1], W_{i} \in \mathbb{R}^{d}\right\},
\end{aligned}
$$

where $\left\{\tilde{\varphi}_{i}\right\}_{i=0}^{r-1}$ are supposed to be linearly independent on $I$ and sufficiently smooth. It is noted that the notation $\tilde{\varphi}_{i}(\tau)$ is referred to $\varphi_{i}(\tau h)$ for all the functions $\varphi_{i}$ throughout this paper. With this definition, we consider another finite-dimensional function space $X_{h}$ such that $\tilde{w}^{\prime} \in Y_{h}$ for any $\tilde{w} \in X_{h}$.

We introduce the idea of the formulation of methods. Find $\tilde{u}(\tau)$ with $\tilde{u}(0)=y_{0}$, satisfying that

$$
\tilde{u}^{\prime}(\tau)=A \tilde{u}(\tau)+\mathcal{P}_{h} g(\tilde{u}(\tau))
$$

where the projection operation $\mathcal{P}_{h}$ is given by (see [38])

$$
\left\langle\tilde{v}(\tau), \mathcal{P}_{h} \tilde{w}(\tau)\right\rangle=\langle\tilde{v}(\tau), \tilde{w}(\tau)\rangle \quad \text { for any } \tilde{v}(\tau) \in Y_{h}
$$


and the inner product $\langle\cdot, \cdot\rangle$ is defined by (see [38]

$$
\left\langle w_{1}, w_{2}\right\rangle=\left\langle w_{1}(\tau), w_{2}(\tau)\right\rangle_{\tau}=\int_{0}^{1} w_{1}(\tau) \cdot w_{2}(\tau) d \tau
$$

With regard to the projection operation $\mathcal{P}_{h}$, we have the following property which is needed in this paper.

Lemma 1 (See [38]) The projection $\mathcal{P}_{h} \tilde{w}$ can be explicitly expressed as

$$
\mathcal{P}_{h} \tilde{w}(\tau)=\left\langle P_{\tau, \sigma}, \tilde{w}(\sigma)\right\rangle_{\sigma}
$$

where

$$
\begin{aligned}
& P_{\tau, \sigma}=\left(\tilde{\varphi}_{0}(\tau), \ldots, \tilde{\varphi}_{r-1}(\tau)\right) \Theta^{-1}\left(\tilde{\varphi}_{0}(\sigma), \ldots, \tilde{\varphi}_{r-1}(\sigma)\right)^{\top}, \\
& \Theta=\left(\left\langle\tilde{\varphi}_{i}(\tau), \tilde{\varphi}_{j}(\tau)\right\rangle\right)_{0 \leq i, j \leq r-1} .
\end{aligned}
$$

When $h$ tends to 0 , the limit of $P_{\tau, \sigma}$ exists. If $P_{\tau, \sigma}$ is computed by a standard orthonormal basis $\left\{\tilde{\psi}_{0}, \ldots, \tilde{\psi}_{r-1}\right\}$ of $Y_{h}$ under the inner product $\langle\cdot, \cdot\rangle$, then $\Theta$ is an identity matrix and $P_{\tau, \sigma}$ has a simpler expression:

$$
P_{\tau, \sigma}=\sum_{i=0}^{r-1} \tilde{\psi}_{i}(\tau) \tilde{\psi}_{i}(\sigma) .
$$

Because $\tilde{u}(\tau)=u(\tau h)$, (7) can be expressed

$$
u^{\prime}(\tau h)=A u(\tau h)+\left\langle P_{\tau, \sigma}, g(u(\sigma h))\right\rangle_{\sigma} .
$$

Applying the variation-of-constants formula (4) to (7), we obtain

$$
\begin{aligned}
& \tilde{u}(\tau)=u(\tau h)=e^{\tau h A} y_{0}+\tau h \int_{0}^{1} e^{(1-\xi) \tau h A}\left\langle P_{\xi \tau, \sigma}, g(u(\sigma h))\right\rangle_{\sigma} d \xi \\
= & e^{\tau h A} y_{0}+\tau h \int_{0}^{1} e^{(1-\xi) \tau h A}\left\langle P_{\xi \tau, \sigma}, g(\tilde{u}(\sigma))\right\rangle_{\sigma} d \xi .
\end{aligned}
$$

Inserting (10) into (11) yields

$$
\begin{aligned}
\tilde{u}(\tau) & =e^{\tau h A} y_{0}+\tau h \int_{0}^{1} e^{(1-\xi) \tau h A} \int_{0}^{1} \sum_{i=0}^{r-1} \tilde{\psi}_{i}(\xi \tau) \tilde{\psi}_{i}(\sigma) g(\tilde{u}(\sigma)) d \sigma d \xi \\
& =e^{\tau h A} y_{0}+\tau h \int_{0}^{1} \sum_{i=0}^{r-1} \int_{0}^{1} e^{(1-\xi) \tau h A} \tilde{\psi}_{i}(\xi \tau) d \xi \tilde{\psi}_{i}(\sigma) g(\tilde{u}(\sigma)) d \sigma .
\end{aligned}
$$

We are now in a position to present the scheme of the novel methods.

Definition 1 An exponential collocation method for solving the system (11) or (3) is defined as follows:

$$
\tilde{u}(\tau)=e^{\tau h A} y_{0}+\tau h \int_{0}^{1} \bar{A}_{\tau, \sigma}(A) g(\tilde{u}(\sigma)) d \sigma, \quad y_{1}=\tilde{u}(1),
$$


where $h$ is a stepsize,

$$
\bar{A}_{\tau, \sigma}(A)=\int_{0}^{1} e^{(1-\xi) \tau h A} P_{\xi \tau, \sigma} d \xi=\sum_{i=0}^{r-1} \int_{0}^{1} e^{(1-\xi) \tau h A} \tilde{\psi}_{i}(\xi \tau) d \xi \tilde{\psi}_{i}(\sigma),
$$

and $\left\{\tilde{\psi}_{0}, \ldots, \tilde{\psi}_{r-1}\right\}$ is a standard orthonormal basis of $Y_{h}$. We denote the method as ECr.

Remark 1 Once the stepsize $h$ is chosen, the method (12) approximates the solution of (11) in the time interval $I_{0}$. Obviously, the obtained result can be considered as the initial condition for a new initial value problem and it can be approximated in the next time interval $I_{1}$. In general, the method can be extended to the approximation of the solution in the interval $[0, T]$.

Remark 2 It can be observed that the ECr method (12) exactly integrates the homogeneous linear system $y^{\prime}=A y$. The scheme (12) can be classified into the category of exponential integrators (which can be thought of as continuous-stage exponential integrators). This is an interesting and important class of numerical methods for first-order ODEs (see, e.g. [30, 31, 32, 33, 34]). In [37], the authors researched a new energy-preserving exponential scheme for the conservative or dissipative system. Here we note that its order is of only two since this scheme combines the ideas of DG and AVF methods. We have proposed a kind of arbitrary-order exponential Fourier collocation methods in [56]. However, those methods cannot preserve energy exactly. Fortunately, we will show that the novel ECr method (12) can be of arbitrarily high order and can exactly or nearly preserve energy, which is different from the existing exponential integrators in the literature. This feature is significant and makes our methods be more efficient and robust.

Remark 3 Consider $M=0$ and $Q=\left(\begin{array}{cc}O_{d_{1} \times d_{1}} & -I_{d_{1} \times d_{1}} \\ I_{d_{1} \times d_{1}} & O_{d_{1} \times d_{1}}\end{array}\right)$, which means that (1) is a Hamiltonian system. In this special case, if we choose $X_{h}$ and $Y_{h}$ as

$$
Y_{h}=\operatorname{span}\left\{\tilde{\varphi}_{0}(\tau), \ldots, \tilde{\varphi}_{r-1}(\tau)\right\}, \quad X_{h}=\operatorname{span}\left\{1, \int_{0}^{\tau} \tilde{\varphi}_{0}(s) d s, \ldots, \int_{0}^{\tau} \tilde{\varphi}_{r-1}(s) d s\right\},
$$

then the ECr method (12) becomes the following energy-preserving Runge-Kutta type collocation methods

$$
\tilde{u}(\tau)=y_{0}+\tau h \int_{0}^{1} \int_{0}^{1} P_{\xi \tau, \sigma} d \xi g(\tilde{u}(\sigma)) d \sigma, \quad y_{1}=\tilde{u}(1),
$$

which yields the functionally-fitted TFE method derived in [38]. Moreover, under the above choices of $M$ and $Q$, if $Y_{h}$ is particularly generated by the shifted Legendre polynomials on [0,1], then the ECr method (12) reduces to the RKEPC method of order $2 r$ given in [23] or $H B V M(\infty, r)$ presented in [6]. Consequently, the ECr method (12) can be regarded as a generalisation of these existing methods in the literature.

\section{Methods for second-order highly oscillatory ODEs}

We consider the following second-order highly oscillatory problems

$$
q^{\prime \prime}(t)-N q^{\prime}(t)+\Omega q(t)=-\nabla U(q(t)), \quad q(0)=q_{0}, \quad q^{\prime}(0)=q_{0}^{\prime}, \quad t \in[0, T],
$$


where $N$ is a symmetric negative semidefinite matrix, $\Omega$ is a symmetric positive semidefinite matrix, and $U: \mathbb{R}^{d} \rightarrow \mathbb{R}$ is a differential function. By introducing $p=q^{\prime}$, (14) can be transformed into

$$
\left(\begin{array}{c}
q \\
p
\end{array}\right)^{\prime}=\left(\begin{array}{cc}
0 & I \\
-I & N
\end{array}\right) \nabla H(q, p)
$$

with

$$
H(q, p)=\frac{1}{2} p^{\top} p+\frac{1}{2} q^{\top} \Omega q+U(q) .
$$

This is exactly the same as the problem (11). Since $N$ is symmetric negative semidefinite, (15) is a dissipative system with the Lyapunov function (16). In the particular case $N=0$, (15) becomes a conservative Hamiltonian system with the first integral (16). This is an important highly oscillatory system which has been investigated by many researchers (see, e.g. [18, 20, 21, 29, 36, 51, 55, 57, 61, 62]).

Applying the ECr method (12) to (15) yields the trigonometric collocation method for secondorder highly oscillatory problems. In particular, for Hamiltonian systems

$$
q^{\prime \prime}(t)+\Omega q(t)=-\nabla U(q(t)),
$$

the case of $N=0$ in (14), the ECr method (12) becomes the following form.

Definition 2 The trigonometric collocation (denoted by TCr) method for (17) is defined as:

$$
\begin{cases}\tilde{q}(\tau)=\phi_{0}(K) q_{0}+\tau h \phi_{1}(K) p_{0}-\tau^{2} h^{2} \int_{0}^{1} \mathcal{A}_{\tau, \sigma}(K) f(\tilde{q}(\sigma)) d \sigma, & q_{1}=\tilde{q}(1) \\ \tilde{p}(\tau)=-\tau h \Omega \phi_{1}(K) q_{0}+\phi_{0}(K) p_{0}-\tau h \int_{0}^{1} \mathcal{B}_{\tau, \sigma}(K) f(\tilde{q}(\sigma)) d \sigma, & p_{1}=\tilde{p}(1)\end{cases}
$$

where $K=\tau^{2} h^{2} \Omega, f(q)=\nabla U(q), \phi_{i}(K):=\sum_{l=0}^{\infty} \frac{(-1)^{l} K^{l}}{(2 l+i) !}$ for $i=0,1, \ldots$, and

$$
\begin{aligned}
& \mathcal{A}_{\tau, \sigma}(K)=\sum_{i=0}^{r-1} \int_{0}^{1}(1-\xi) \phi_{1}\left((1-\xi)^{2} K\right) \tilde{\psi}_{i}(\xi \tau) d \xi \tilde{\psi}_{i}(\sigma), \\
& \mathcal{B}_{\tau, \sigma}(K)=\sum_{i=0}^{r-1} \int_{0}^{1} \phi_{0}\left((1-\xi)^{2} K\right) \tilde{\psi}_{i}(\xi \tau) d \xi \tilde{\psi}_{i}(\sigma) .
\end{aligned}
$$

Remark 4 In [50], the authors developed and researched a novel type of trigonometric Fourier collocation methods for second-order ODEs $q^{\prime \prime}(t)+M q(t)=f(q(t))$. However, as shown in [50], those methods cannot preserve the energy exactly. From the analysis to be presented in this paper, it turns out that the trigonometric collocation scheme (18) developed here can attain arbitrary algebraic order and can exactly or nearly preserve the energy of (16).

Remark 5 It is remarked that the multi-frequency highly oscillatory Hamiltonian system (5) is a kind of second-order system $q^{\prime \prime}(t)+\bar{M}^{-1} \bar{K} q(t)=-\bar{M}^{-1} \nabla U(q(t))$ and applying the ECr method (12) to it leads to the TCr method (18) with $K=\tau^{2} h^{2} \bar{M}^{-1} \bar{K}$ and $f(q)=\bar{M}^{-1} \nabla U(q)$. 
In the special case of $N=0$ and $\Omega=0$, the system (14) reduces to the conventional second-order ODEs

$$
q^{\prime \prime}(t)=-\nabla U(q(t)), \quad q(0)=q_{0}, \quad q^{\prime}(0)=q_{0}^{\prime}, \quad t \in[0, T] .
$$

Then the TCr method has the following form.

Definition 3 A TCr method for solving (20) is defined as

$$
\begin{cases}\tilde{q}(\tau)=q_{0}+\tau h p_{0}-\tau^{2} h^{2} \int_{0}^{1} \overline{\mathcal{A}}_{\tau, \sigma} \nabla U(\tilde{q}(\sigma)) d \sigma, & q_{1}=\tilde{q}(1), \\ \tilde{p}(\tau)=p_{0}-\tau h \int_{0}^{1} \overline{\mathcal{B}}_{\tau, \sigma} \nabla U(\tilde{q}(\sigma)) d \sigma, & p_{1}=\tilde{p}(1),\end{cases}
$$

where

$$
\overline{\mathcal{A}}_{\tau, \sigma}=\sum_{i=0}^{r-1} \int_{0}^{1}(1-\xi) \tilde{\psi}_{i}(\xi \tau) d \xi \tilde{\psi}_{i}(\sigma), \overline{\mathcal{B}}_{\tau, \sigma}=\sum_{i=0}^{r-1} \int_{0}^{1} \tilde{\psi}_{i}(\xi \tau) d \xi \tilde{\psi}_{i}(\sigma) .
$$

This scheme looks like a continuous-stage RKN method, and is denoted by RKNCr in this paper.

\section{$4 \quad$ Energy-preserving analysis}

In this section, we analyse the energy-preserving property of the ECr methods.

Theorem 1 If $Q$ is skew symmetric and $\tilde{u}(\tau) \in X_{h}$, the first integral $H$ (2) of the conservative system (11) can be preserved exactly by the ECr method (12): i.e., $H\left(y_{1}\right)=H\left(y_{0}\right)$. If $\tilde{u}(\tau) \notin X_{h}$, the ECr method (12) approximately preserves the energy $H$ with the following accuracy $H\left(y_{1}\right)=$ $H\left(y_{0}\right)+\mathcal{O}\left(h^{2 r+1}\right)$.

Proof We begin with the first part of this proof under the assumption that $Q$ is skew symmetric and $\tilde{u}(\tau) \in X_{h}$. From $\tilde{u}(\tau) \in X_{h}$, it follows that $\tilde{u}^{\prime}(\tau) \in Y_{h}$ and $Q^{-1} \tilde{u}^{\prime}(\tau) \in Y_{h}$. Then, in the light of (8), we obtain

$$
\begin{aligned}
\int_{0}^{1} \tilde{u}^{\prime}(\tau)^{\top}\left(Q^{-1}\right)^{\top} \tilde{u}^{\prime}(\tau) d \tau & =\int_{0}^{1} \tilde{u}^{\prime}(\tau)^{\top}\left(Q^{-1}\right)^{\top}\left(A \tilde{u}(\tau)+\mathcal{P}_{h} g(\tilde{u}(\tau))\right) d \tau \\
& =\int_{0}^{1} \tilde{u}^{\prime}(\tau)^{\top}\left(Q^{-1}\right)^{\top}(A \tilde{u}(\tau)+g(\tilde{u}(\tau))) d \tau
\end{aligned}
$$

Here $Q$ is skew symmetric, so does $Q^{-1}$. We then have

$$
0=\int_{0}^{1} \tilde{u}^{\prime}(\tau)^{\top}\left(Q^{-1}\right)^{\top} \tilde{u}^{\prime}(\tau) d \tau=-\int_{0}^{1} \tilde{u}^{\prime}(\tau)^{\top} Q^{-1}(A \tilde{u}(\tau)+g(\tilde{u}(\tau))) d \tau
$$

On the other hand, it is clear that

$$
H\left(y_{1}\right)-H\left(y_{0}\right)=\int_{0}^{1} \frac{d}{d \tau} H(\tilde{u}(\tau)) d \tau=h \int_{0}^{1} \tilde{u}^{\prime}(\tau)^{\top} \nabla H(\tilde{u}(\tau)) d \tau .
$$

It follows from (11) and (3) that

$$
\nabla H(\tilde{u}(\tau))=Q^{-1}(A \tilde{u}(\tau)+g(\tilde{u}(\tau))) .
$$


Therefore, we obtain

$$
H\left(y_{1}\right)-H\left(y_{0}\right)=h \int_{0}^{1} \tilde{u}^{\prime}(\tau)^{\top} Q^{-1}(A \tilde{u}(\tau)+g(\tilde{u}(\tau))) d \tau=h \cdot 0=0 .
$$

We next prove the second part of this theorem under the assumption that $\tilde{u}(\tau) \notin X_{h}$. With the above analysis for the first part of the proof, we have

$$
\begin{aligned}
& H\left(y_{1}\right)-H\left(y_{0}\right)=h \int_{0}^{1} \tilde{u}^{\prime}(\tau)^{\top} Q^{-1}(A \tilde{u}(\tau)+g(\tilde{u}(\tau))) d \tau \\
= & h \int_{0}^{1} \tilde{u}^{\prime}(\tau)^{\top} Q^{-1}\left(A \tilde{u}(\tau)+\mathcal{P}_{h} g(\tilde{u}(\tau))+g(\tilde{u}(\tau))-\mathcal{P}_{h} g(\tilde{u}(\tau))\right) d \tau \\
= & -h \int_{0}^{1} \tilde{u}^{\prime}(\tau)^{\top}\left(Q^{-1}\right)^{\top} \tilde{u}^{\prime}(\tau) d \tau+h \int_{0}^{1} \tilde{u}^{\prime}(\tau)^{\top} Q^{-1}\left(g(\tilde{u}(\tau))-\mathcal{P}_{h} g(\tilde{u}(\tau))\right) d \tau \\
= & h \int_{0}^{1} \tilde{u}^{\prime}(\tau)^{\top} Q^{-1}\left(g(\tilde{u}(\tau))-\mathcal{P}_{h} g(\tilde{u}(\tau))\right) d \tau .
\end{aligned}
$$

Concerning Lemma 3.4 presented in [38] and Lemma 2 proved in Section [6, one has $\tilde{u}^{\prime}(\tau)=$ $\mathcal{P}_{h} \tilde{u}^{\prime}(\tau)+\mathcal{O}\left(h^{r}\right)$. Therefore, one arrives at

$$
\begin{aligned}
H\left(y_{1}\right)-H\left(y_{0}\right) & =h \int_{0}^{1}\left(\mathcal{P}_{h} \tilde{u}^{\prime}(\tau)+\mathcal{O}\left(h^{r}\right)\right)^{\top} Q^{-1}\left(g(\tilde{u}(\tau))-\mathcal{P}_{h} g(\tilde{u}(\tau))\right) d \tau \\
& =h \int_{0}^{1}\left(\mathcal{P}_{h} \tilde{u}^{\prime}(\tau)\right)^{\top} Q^{-1}\left(g(\tilde{u}(\tau))-\mathcal{P}_{h} g(\tilde{u}(\tau))\right) d \tau+\mathcal{O}\left(h^{2 r+1}\right) \\
& =h \int_{0}^{1}\left(\mathcal{P}_{h} \tilde{u}^{\prime}(\tau)\right)^{\top} Q^{-1}(g(\tilde{u}(\tau))-g(\tilde{u}(\tau))) d \tau+\mathcal{O}\left(h^{2 r+1}\right)=\mathcal{O}\left(h^{2 r+1}\right),
\end{aligned}
$$

where the result (28) is used.

The proof is complete.

Theorem 2 If $Q$ is negative semidefinite and $\tilde{u}(\tau) \in X_{h}$, then the Lyapunov function $H$ given by (2) of the dissipative system (11) can be preserved by the ECr method (12); i.e., $H\left(y_{1}\right) \leq H\left(y_{0}\right)$. If $\tilde{u}(\tau) \notin X_{h}$, it is true that $H\left(y_{1}\right) \leq H\left(y_{0}\right)+\mathcal{O}\left(h^{2 r+1}\right)$.

Proof According to the fact that $\int_{0}^{1} \tilde{u}^{\prime}(\tau)^{\top} Q^{-1} \tilde{u}^{\prime}(\tau) d \tau \leq 0$, this theorem can be proved in a similar way to the proof of Theorem 1

\section{The existence, uniqueness and smoothness}

In this section, we focus on the study of the existence and uniqueness of $\tilde{u}(\tau)$ associated with the ECr method (12).

According to the Lemma 3.1 given in [32], it is easily verified that the coefficients $e^{\tau h A}$ and $\bar{A}_{\tau, \sigma}(A)$ of our methods for $0 \leq \tau \leq 1$ and $0 \leq \sigma \leq 1$ are uniformly bounded. We begin with assuming that

$$
M_{k}=\max _{\tau, \sigma, h \in[0,1]}\left\|\frac{\partial^{k} \bar{A}_{\tau, \sigma}}{\partial h^{k}}\right\|, \quad C_{k}=\max _{\tau, h \in[0,1]}\left\|\frac{\partial^{k} e^{\tau h A}}{\partial h^{k}} y_{0}\right\|, \quad k=0,1, \ldots
$$


Furthermore, the $n$ th-order derivative of $g$ at $y$ is denoted by $g^{(n)}(y)$. We then have the following result about the existence and uniqueness of our methods.

Theorem 3 Let $B\left(\bar{y}_{0}, R\right)=\left\{y \in \mathbb{R}^{d}:\left\|y-\bar{y}_{0}\right\| \leq R\right\}$ and

$$
D_{n}=\max _{y \in B\left(\bar{y}_{0}, R\right)}\left\|g^{(n)}(y)\right\|, n=0,1, \ldots,
$$

where $R$ is a positive constant, $\bar{y}_{0}=e^{\tau h A} y_{0},\|\cdot\|=\|\cdot\|_{\infty}$ is the maximum norm for vectors in $\mathbb{R}^{d}$ or the corresponding induced norm for the multilinear maps $g^{(n)}(y)$. If $h$ satisfies

$$
0 \leq h \leq \kappa<\min \left\{\frac{1}{M_{0} D_{1}}, \frac{R}{M_{0} D_{0}}, 1\right\},
$$

then the ECr method (12) has a unique solution $\tilde{u}(\tau)$ which is smoothly dependent on $h$.

Proof $\operatorname{Set} \tilde{u}_{0}(\tau)=\bar{y}_{0}$ and define

$$
\tilde{u}_{n+1}(\tau)=e^{\tau h A} y_{0}+\tau h \int_{0}^{1} \bar{A}_{\tau, \sigma}(A) g\left(\tilde{u}_{n}(\sigma)\right) d \sigma, \quad n=0,1, \ldots,
$$

which leads to a function series $\left\{\tilde{u}_{n}(\tau)\right\}_{n=0}^{\infty}$. We note that $\lim _{n \rightarrow \infty} \tilde{u}_{n}(\tau)$ is a solution of the $\mathrm{TCr}$ method (12) if $\left\{\tilde{u}_{n}(\tau)\right\}_{n=0}^{\infty}$ is uniformly convergent, which will be shown by proving the uniform convergence of the infinite series $\sum_{n=0}^{\infty}\left(\tilde{u}_{n+1}(\tau)-\tilde{u}_{n}(\tau)\right)$.

By induction and according to (23) and (24), we get $\left\|\tilde{u}_{n}(\tau)-\bar{y}_{0}\right\| \leq R$ for $n=0,1, \ldots$ It then follows from (24) that

$$
\begin{aligned}
& \left\|\tilde{u}_{n+1}(\tau)-\tilde{u}_{n}(\tau)\right\| \leq \tau h \int_{0}^{1} M_{0} D_{1}\left\|\tilde{u}_{n}(\sigma)-\tilde{u}_{n-1}(\sigma)\right\| d \sigma \\
& \leq h \int_{0}^{1} M_{0} D_{1}\left\|\tilde{u}_{n}(\sigma)-\tilde{u}_{n-1}(\sigma)\right\| d \sigma \leq \beta\left\|\tilde{u}_{n}-\tilde{u}_{n-1}\right\|_{c}, \quad \beta=\kappa M_{0} D_{1},
\end{aligned}
$$

where $\|\cdot\|_{c}$ is the maximum norm for continuous functions defined as $\|w\|_{c}=\max _{\tau \in[0,1]}\|w(\tau)\|$ for a continuous $\mathbb{R}^{d}$-valued function $w$ on $[0,1]$. Hence, we obtain

$$
\left\|\tilde{u}_{n+1}-\tilde{u}_{n}\right\|_{c} \leq \beta\left\|\tilde{u}_{n}-\tilde{u}_{n-1}\right\|_{c}
$$

and

$$
\left\|\tilde{u}_{n+1}-\tilde{u}_{n}\right\|_{c} \leq \beta^{n}\left\|\tilde{u}_{1}-y_{0}\right\|_{c} \leq \beta^{n} R, \quad n=0,1, \ldots
$$

From Weierstrass $M$-test and the fact that $\beta<1$, it immediately follows that $\sum_{n=0}^{\infty}\left(\tilde{u}_{n+1}(\tau)-\right.$ $\left.\tilde{u}_{n}(\tau)\right)$ is uniformly convergent

If the ECr method (12) has another solution $\tilde{v}(\tau)$, we then obtain the following inequalities

$$
\|\tilde{u}(\tau)-\tilde{v}(\tau)\| \leq h \int_{0}^{1}\left\|\bar{A}_{\tau, \sigma}(A)(g(\tilde{u}(\sigma))-g(\tilde{v}(\sigma)))\right\| d \sigma \leq \beta\|\tilde{u}-\tilde{v}\|_{c},
$$

and $\|\tilde{u}-\tilde{v}\|_{c} \leq \beta\|\tilde{u}-\tilde{v}\|_{c}$. This yields $\|\tilde{u}-\tilde{v}\|_{c}=0$ and $\tilde{u}(\tau) \equiv \tilde{v}(\tau)$. The existence and uniqueness have been proved. 
With respect to the result that $\tilde{u}(\tau)$ is smoothly dependent of $h$, since each $\tilde{u}_{n}(\tau)$ is a smooth function of $h$, we need only to prove that the series $\left\{\frac{\partial^{k} \tilde{u}_{n}}{\partial h^{k}}(\tau)\right\}_{n=0}^{\infty}$ is uniformly convergent for $k \geq 1$. Differentiating (24) with respect to $h$ gives

$$
\begin{aligned}
\frac{\partial \tilde{u}_{n+1}}{\partial h}(\tau)= & \tau A e^{\tau h A} y_{0}+\tau \int_{0}^{1}\left(\bar{A}_{\tau, \sigma}(A)+h \frac{\partial \bar{A}_{\tau, \sigma}}{\partial h}\right) g\left(\tilde{u}_{n}(\sigma)\right) d \sigma \\
& +\tau h \int_{0}^{1} \bar{A}_{\tau, \sigma}(A) g^{(1)}\left(\tilde{u}_{n}(\sigma)\right) \frac{\partial \tilde{u}_{n}}{\partial h}(\sigma) d \sigma
\end{aligned}
$$

which yields that

$$
\left\|\frac{\partial \tilde{u}_{n+1}}{\partial h}\right\|_{c} \leq \alpha+\beta\left\|\frac{\partial \tilde{u}_{n}}{\partial h}\right\|_{c}, \quad \alpha=C_{1}+\left(M_{0}+\kappa M_{1}\right) D_{0} .
$$

By induction, it is easy to show that $\left\{\frac{\partial \tilde{u}_{n}}{\partial h}(\tau)\right\}_{n=0}^{\infty}$ is uniformly bounded:

$$
\left\|\frac{\partial \tilde{u}_{n}}{\partial h}\right\|_{c} \leq \alpha\left(1+\beta+\ldots+\beta^{n-1}\right) \leq \frac{\alpha}{1-\beta}=C^{*}, \quad n=0,1, \ldots
$$

It follows from (25)-(26) that

$$
\begin{aligned}
& \left\|\frac{\partial \tilde{u}_{n+1}}{\partial h}-\frac{\partial \tilde{u}_{n}}{\partial h}\right\|_{c} \leq \tau \int_{0}^{1}\left(M_{0}+h M_{1}\right)\left\|g\left(\tilde{u}_{n}(\sigma)\right)-g\left(\tilde{u}_{n-1}(\sigma)\right)\right\| d \sigma \\
& +\tau h \int_{0}^{1} M_{0}\left(\left\|\left(g^{(1)}\left(\tilde{u}_{n}(\sigma)\right)-g^{(1)}\left(\tilde{u}_{n-1}(\sigma)\right)\right) \frac{\partial \tilde{u}_{n}}{\partial h}(\sigma)\right\|\right. \\
& \left.+\left\|g^{(1)}\left(\tilde{u}_{n-1}(\sigma)\right)\left(\frac{\partial \tilde{u}_{n}}{\partial h}(\sigma)-\frac{\partial \tilde{u}_{n-1}}{\partial h}(\sigma)\right)\right\|\right) d \sigma \leq \gamma \beta^{n-1}+\beta\left\|\frac{\partial \tilde{u}_{n}}{\partial h}-\frac{\partial \tilde{u}_{n-1}}{\partial h}\right\|_{c},
\end{aligned}
$$

where $\gamma=\left(M_{0} D_{1}+\kappa M_{1} D_{1}+\kappa M_{0} L_{2} C^{*}\right) R$, and $L_{2}$ is a constant satisfying

$$
\left\|g^{(1)}(y)-g^{(1)}(z)\right\| \leq L_{2}\|y-z\|, \quad \text { for } y, z \in B\left(\bar{y}_{0}, R\right) .
$$

Therefore, the following result is obtained by induction

$$
\left\|\frac{\partial \tilde{u}_{n+1}}{\partial h}-\frac{\partial \tilde{u}_{n}}{\partial h}\right\|_{c} \leq n \gamma \beta^{n-1}+\beta^{n} C^{*}, \quad n=1,2, \ldots
$$

This shows the uniform convergence of $\sum_{n=0}^{\infty}\left(\frac{\partial \tilde{u}_{n+1}}{\partial h}(\tau)-\frac{\partial \tilde{u}_{n}}{\partial h}(\tau)\right)$ and then $\left\{\frac{\partial \tilde{u}_{n}}{\partial h}(\tau)\right\}_{n=0}^{\infty}$ is uniformly convergent.

Likewise, it can be shown that other function series $\left\{\frac{\partial^{k} \tilde{u}_{n}}{\partial h^{k}}(\tau)\right\}_{n=0}^{\infty}$ for $k \geq 2$ are uniformly convergent as well. Therefore, $\tilde{u}(\tau)$ is smoothly dependent on $h$.

\section{Algebraic order}

In this section, we analyse the algebraic order of the ECr method (12). To express the dependence of the solutions of $y^{\prime}(t)=A y(t)+g(y(t))$ on the initial values, we denote by $y(\cdot, \tilde{t}, \tilde{y})$ the solution 
satisfying the initial condition $y(\tilde{t}, \tilde{t}, \tilde{y})=\tilde{y}$ for any given $\tilde{t} \in[0, h]$ and set $\Phi(s, \tilde{t}, \tilde{y})=\frac{\partial y(s, \tilde{t}, \tilde{y})}{\partial \tilde{y}}$. Recalling the elementary theory of ODEs, we have the following standard result

$$
\frac{\partial y(s, \tilde{t}, \tilde{y})}{\partial \tilde{t}}=-\Phi(s, \tilde{t}, \tilde{y})(A \tilde{y}+g(\tilde{y}))
$$

In this section, for convenience, an $h$-dependent function $w(\tau)$ is called as regular if it can be expanded as $w(\tau)=\sum_{n=0}^{r-1} w^{[n]}(\tau) h^{n}+\mathcal{O}\left(h^{r}\right)$, where $w^{[n]}(\tau)=\left.\frac{1}{n !} \frac{\partial^{n} w(\tau)}{\partial h^{n}}\right|_{h=0}$ is a vector-valued function with polynomial entries of degrees $\leq n$.

It can be deduced from Proposition 3.3 in 38 that $P_{\tau, \sigma}$ is regular. Moreover, we can prove the following result.

Lemma 2 The ECr method (12) gives a regular $h$-dependent function $\tilde{u}(\tau)$.

$\underline{\text { Proof }}$ By the result given in $\left[38\right.$, we know that $P_{\tau, \sigma}$ can be smoothly extended to $h=0$ by setting $\left.\bar{P}_{\tau, \sigma}\right|_{h=0}=\lim _{h \rightarrow 0} P_{\tau, \sigma}(h)$. Furthermore, it follows from Theorem 3 that $\tilde{u}(\tau)$ is smoothly dependent on $h$. Therefore, $\tilde{u}(\tau)$ and $\bar{A}_{\tau, \sigma}(A)$ can be expanded with respect to $h$ at zero as follows:

$$
\tilde{u}(\tau)=\sum_{m=0}^{r-1} \tilde{u}^{[m]}(\tau) h^{m}+\mathcal{O}\left(h^{r}\right), \quad \bar{A}_{\tau, \sigma}(A)=\sum_{m=0}^{r-1} \bar{A}_{\tau, \sigma}^{[m]}(A) h^{m}+\mathcal{O}\left(h^{r}\right) .
$$

Then let $\delta=\tilde{u}(\sigma)-y_{0}$ and we have

$$
\delta=\tilde{u}^{[0]}(\sigma)-y_{0}+\mathcal{O}(h)=y_{0}-y_{0}+\mathcal{O}(h)=\mathcal{O}(h) .
$$

We expand $f(\tilde{u}(\sigma))$ at $y_{0}$ and insert the above equalities into the first equation of the ECr method (12). This manipulation yields

$$
\begin{aligned}
& \sum_{m=0}^{r-1} \tilde{u}^{[m]}(\tau) h^{m}=\sum_{m=0}^{r-1} \frac{\tau^{m} A^{m} y_{0}}{m !} h^{m} \\
& +\tau h \int_{0}^{1} \sum_{k=0}^{r-1} \bar{A}_{\tau, \sigma}^{[k]}(A) h^{k} \sum_{n=0}^{r-1} \frac{1}{n !} g^{(n)}\left(y_{0}\right)(\underbrace{\delta, \ldots, \delta}_{n-\text { fold }}) d \sigma+\mathcal{O}\left(h^{r}\right) .
\end{aligned}
$$

In order to show that $\tilde{u}(\tau)$ is regular, we need only to prove that

$$
\tilde{u}^{[m]}(\tau) \in P_{m}^{d}=\underbrace{P_{m}([0,1]) \times \ldots \times P_{m}([0,1])}_{d-\text { fold }} \text { for } m=0,1, \ldots, r-1,
$$

where $P_{m}([0,1])$ consists of polynomials of degrees $\leq m$ on $[0,1]$. This can be confirmed by induction as follows.

Firstly, it is clear that $\tilde{u}^{[0]}(\tau)=y_{0} \in P_{0}^{d}$. We assume that $\tilde{u}^{[n]}(\tau) \in P_{n}^{d}$ for $n=0,1, \ldots, m$. Comparing the coefficients of $h^{m+1}$ on both sides of (27) and using (13) lead to

$$
\begin{aligned}
& \tilde{u}^{[m+1]}(\tau)=\frac{\tau^{m+1} A^{m+1}}{(m+1) !} y_{0}+\sum_{k+n=m} \tau \int_{0}^{1} \bar{A}_{\tau, \sigma}^{[k]}(A) h_{n}(\sigma) d \sigma \\
& =\frac{\tau^{m+1} A^{m+1}}{(m+1) !} y_{0}+\sum_{k+n=m} \tau \int_{0}^{1} \int_{0}^{1}\left[e^{(1-\xi) \tau h A} P_{\xi \tau, \sigma}\right]^{[k]} h_{n}(\sigma) d \sigma d \xi, \quad h_{n}(\sigma) \in P_{n}^{d} .
\end{aligned}
$$


Since $P_{\xi \tau, \sigma}$ is regular, it is easy to check that $e^{(1-\xi) \tau h A} P_{\xi \tau, \sigma}$ is also regular. Thus, under the condition $k+n=m$, we have

$$
\int_{0}^{1}\left[e^{(1-\xi) \tau h A} P_{\xi \tau, \sigma}\right]^{[k]} h_{n}(\sigma) d \sigma:=\check{p}_{m}^{k}(\xi \tau) \in P_{m}^{d}([0,1]) .
$$

Then, the above result can be simplified as

$$
\begin{aligned}
\tilde{u}^{[m+1]}(\tau) & =\frac{\tau^{m+1} A^{m+1}}{(m+1) !} y_{0}+\sum_{k+n=m} \tau \int_{0}^{1} \check{p}_{m}^{k}(\xi \tau) d \xi \\
& =\frac{\tau^{m+1} A^{m+1}}{(m+1) !} y_{0}+\sum_{k+n=m} \int_{0}^{\tau} \check{p}_{m}^{k}(\alpha) d \alpha \in P_{m+1}^{d} .
\end{aligned}
$$

According to Lemma 3.4 presented in 38 and the above lemma, we get

$$
\mathcal{P}_{h} g(\tilde{u}(\tau))-g(\tilde{u}(\tau))=\mathcal{O}\left(h^{r}\right)
$$

which will be used in the analysis of algebraic order. We are now ready to present the result about the algebraic order of the ECr method (12).

Theorem 4 About the stage order and order of the ECr method (12), we have

$$
\begin{aligned}
& \tilde{u}(\tau)-y\left(t_{0}+\tau h\right)=\mathcal{O}\left(h^{r+1}\right), \quad 0<\tau<1, \\
& \tilde{u}(1)-y\left(t_{0}+h\right)=\mathcal{O}\left(h^{2 r+1}\right) .
\end{aligned}
$$

Proof According to the previous preliminaries, we obtain

$$
\begin{aligned}
& \tilde{u}(\tau)-y\left(t_{0}+\tau h\right)=y\left(t_{0}+\tau h, t_{0}+\tau h, \tilde{u}(\tau)\right)-y\left(t_{0}+\tau h, t_{0}, y_{0}\right) \\
& =\int_{0}^{\tau} \frac{d}{d \alpha} y\left(t_{0}+\tau h, t_{0}+\alpha h, \tilde{u}(\alpha)\right) d \alpha \\
& =\int_{0}^{\tau}\left(h \frac{\partial y}{\partial \tilde{t}}\left(t_{0}+\tau h, t_{0}+\alpha h, \tilde{u}(\alpha)\right)+\frac{\partial y}{\partial \tilde{y}}\left(t_{0}+\tau h, t_{0}+\alpha h, \tilde{u}(\alpha)\right) h \tilde{u}^{\prime}(\alpha)\right) d \alpha \\
& =\int_{0}^{\tau}\left(-h \frac{\partial y}{\partial \tilde{y}}\left(t_{0}+\tau h, t_{0}+\alpha h, \tilde{u}(\alpha)\right)(A \tilde{u}(\alpha)+g(\tilde{u}(\alpha)))\right. \\
& \left.\quad+\frac{\partial y}{\partial \tilde{y}}\left(t_{0}+\tau h, t_{0}+\alpha h, \tilde{u}(\alpha)\right)\left(h A \tilde{u}(\alpha)+h\left\langle P_{\tau, \sigma}, g(\tilde{u}(\alpha))\right\rangle_{\alpha}\right)\right) d \alpha \\
& =-h \int_{0}^{\tau} \Phi^{\tau}(\alpha)\left(g(\tilde{u}(\alpha))-\mathcal{P}_{h}(g \circ \tilde{u})(\alpha)\right) d \alpha=\mathcal{O}\left(h^{r+1}\right),
\end{aligned}
$$

where $\Phi^{\tau}(\alpha)=\frac{\partial y}{\partial \tilde{y}}\left(t_{0}+\tau h, t_{0}+\alpha h, \tilde{u}(\alpha)\right)$. Letting $\tau=1$ in (29) yields

$$
\tilde{u}(1)-y\left(t_{0}+h\right)=-h \int_{0}^{1} \Phi^{1}(\alpha)\left(g(\tilde{u}(\alpha))-\mathcal{P}_{h}(g \circ \tilde{u})(\alpha)\right) d \alpha .
$$

We partition the matrix-valued function $\Phi^{1}(\alpha)$ as $\Phi^{1}(\alpha)=\left(\Phi_{1}^{1}(\alpha), \ldots, \Phi_{d}^{1}(\alpha)\right)^{\top}$. It follows from Lemma 2 that

$$
\Phi_{i}^{1}(\alpha)=\mathcal{P}_{h} \Phi_{i}^{1}(\alpha)+\mathcal{O}\left(h^{r}\right), \quad i=1, \ldots, d .
$$


On the other hand, we have

$$
\int_{0}^{1}\left(\mathcal{P}_{h} \Phi_{i}^{1}(\alpha)\right)^{\top} g(\tilde{u}(\alpha)) d \alpha=\int_{0}^{1}\left(\mathcal{P}_{h} \Phi_{i}^{1}(\alpha)\right)^{\top} \mathcal{P}_{h}(g \circ \tilde{u})(\alpha) d \alpha, \quad i=1, \ldots, d .
$$

Therefore, from (30), (31) and (32), it follows that

$$
\begin{aligned}
& \tilde{u}(1)-y\left(t_{0}+h\right)=-h \int_{0}^{1}\left(\left(\begin{array}{c}
\left(\mathcal{P}_{h} \Phi_{1}^{1}(\alpha)\right)^{\top} \\
\vdots \\
\left(\mathcal{P}_{h} \Phi_{d}^{1}(\alpha)\right)^{\top}
\end{array}\right)+\mathcal{O}\left(h^{r}\right)\right)\left(g(\tilde{u}(\alpha))-\mathcal{P}_{h}(g \circ \tilde{u})(\alpha)\right) d \alpha \\
= & -h \int_{0}^{1}\left(\begin{array}{c}
\left(\mathcal{P}_{h} \Phi_{1}^{1}(\alpha)\right)^{\top}\left(g(\tilde{u}(\alpha))-\mathcal{P}_{h}(g \circ \tilde{u})(\alpha)\right) \\
\vdots \\
\left(\mathcal{P}_{h} \Phi_{d}^{1}(\alpha)\right)^{\top}\left(g(\tilde{u}(\alpha))-\mathcal{P}_{h}(g \circ \tilde{u})(\alpha)\right)
\end{array}\right) d \alpha-h \int_{0}^{1} \mathcal{O}\left(h^{r}\right) \times \mathcal{O}\left(h^{r}\right) d \alpha \\
= & 0+\mathcal{O}\left(h^{2 r+1}\right)=\mathcal{O}\left(h^{2 r+1}\right) .
\end{aligned}
$$

\section{Application in stiff gradient systems}

When the matrix $Q$ in (1) is identity matrix, then the system (1) is a stiff gradient system as follows:

$$
y^{\prime}=-\nabla U(y), \quad y(0)=y_{0} \in \mathbb{R}^{d}, \quad t \in[0, T],
$$

where the potential $U$ has the form

$$
U(y)=\frac{1}{2} y^{\top} M y+V(y) .
$$

Such problems arise from the spatial discretisation of Allen-Cahn and Cahn-Hilliard PDEs (see, e.g. [2]). Along every exact solution, it is true that

$$
\frac{d}{d t} U(y(t))=\nabla U(y(t))^{\top} y^{\prime}(t)=-y^{\prime}(t)^{\top} y^{\prime}(t) \leq 0,
$$

which implies that $U(y(t))$ is monotonically decreasing.

For solving this stiff gradient system, it follows from Theorem 2 that our practical ECr method (40) is unconditionally energy-diminishing. For a quadratic potential (i.e., $V(y)=0$ in (34)), the numerical solution of our method is given by

$$
y_{1}=R(-h A) y_{0}=e^{-h A} y_{0} .
$$

The importance of the damping property $|R(\infty)|<1$ for the approximation properties of RungeKutta methods has been studied and well understood in [39, 40] for solving semilinear parabolic equations. The role of the condition $|R(\infty)|<1$ in the approximation of stiff differential equations has been researched in Chapter VI of [26]. It has been shown in [24] that for each Runge-Kutta method the energy decreases once the stepsize satisfies some conditions. Discrete-gradient methods, AVF methods and AVF collocation methods derived in 24] are unconditionally energy-diminishing 
methods but they show no damping for very stiff gradient systems. However, it is clear that our methods are unconditionally energy-diminishing methods and they have

$$
|R(\infty)|=\left|e^{-\infty}\right|=0 .
$$

This implies that our methods are strongly damped even for very stiff gradient systems and this is a significant feature.

\section{Practical examples of the new methods}

In this section, we present the practical examples of the new methods. By the choice of $\tilde{\varphi}_{k}(\tau)=$ $(\tau h)^{k}$ for $k=0,1, \ldots, r-1$ and using the Gram-Schmide process, we obtain the standard orthonormal basis of $Y_{h}$ as

$$
\hat{p}_{j}(\tau)=(-1)^{j} \sqrt{2 j+1} \sum_{k=0}^{j}\left(\begin{array}{l}
j \\
k
\end{array}\right)\left(\begin{array}{c}
j+k \\
k
\end{array}\right)(-\tau)^{k}, \quad j=0,1, \ldots, r-1, \quad \tau \in[0,1],
$$

which are the shifted Legendre polynomials on $[0,1]$. Therefore, $P_{\tau, \sigma}$ can be determined by its limit as follows $P_{\tau, \sigma}=\lim _{h \rightarrow 0} P_{\tau, \sigma}=\sum_{i=0}^{r-1} \hat{p}_{i}(\tau) \hat{p}_{i}(\sigma)$,

\subsection{An example of ECr methods}

For the ECr method (12), we need to calculate $\bar{A}_{\tau, \sigma}(A)$ appearing in the methods. It follows form (13) that

$$
\begin{aligned}
& \bar{A}_{\tau, \sigma}(A)=\int_{0}^{1} e^{(1-\xi) \tau h A} P_{\xi \tau, \sigma} d \xi=\sum_{i=0}^{r-1} \int_{0}^{1} e^{(1-\xi) \tau h A} \hat{p}_{i}(\xi \tau) d \xi \hat{p}_{i}(\sigma) \\
= & \sum_{i=0}^{r-1} \int_{0}^{1} e^{(1-\xi) \tau h A}(-1)^{i} \sqrt{2 i+1} \sum_{k=0}^{i}\left(\begin{array}{c}
i \\
k
\end{array}\right)\left(\begin{array}{c}
i+k \\
k
\end{array}\right)(-\xi \tau)^{k} d \xi \hat{p}_{i}(\sigma) \\
= & \sum_{i=0}^{r-1} \sqrt{2 i+1} \sum_{k=0}^{i}(-1)^{i+k} \frac{(i+k) !}{k !(i-k) !} \bar{\varphi}_{k+1}(\tau h A) \hat{p}_{i}(\sigma) .
\end{aligned}
$$

Here the $\bar{\varphi}$-functions (see, e.g. [30, 32, 33, 34]) are defined by:

$$
\bar{\varphi}_{0}(z)=e^{z}, \quad \bar{\varphi}_{k}(z)=\int_{0}^{1} e^{(1-\sigma) z} \frac{\sigma^{k-1}}{(k-1) !} d \sigma, \quad k=1,2, \ldots
$$

It is noted that a number of approaches have been developed which work with the application of the $\varphi$-functions on a vector (see [1, 28, 33, for example). 


\subsection{An example of $\mathrm{TCr}$ methods}

For the TCr method (18) of solving $q^{\prime \prime}(t)+\Omega q(t)=-\nabla U(q(t))$, we need to compute $\mathcal{A}_{\tau, \sigma}$ and $\mathcal{B}_{1, \sigma}$. It follows from (19) that

$$
\begin{aligned}
& \mathcal{A}_{\tau, \sigma}(K)=\sum_{j=0}^{r-1} \int_{0}^{1}(1-\xi) \phi_{1}\left((1-\xi)^{2} K\right) \hat{p}_{j}(\xi \tau) d \xi \hat{p}_{j}(\sigma) \\
= & \sum_{j=0}^{r-1} \sqrt{2 j+1} \sum_{l=0}^{\infty}(-1)^{j} \sum_{k=0}^{j}\left(\begin{array}{c}
j \\
k
\end{array}\right)\left(\begin{array}{c}
j+k \\
k
\end{array}\right) \int_{0}^{1}(-\xi)^{k}(1-\xi)^{2 l+1} d \xi \frac{(-1)^{l} K^{l}}{(2 l+1) !} \tau^{k} \hat{p}_{j}(\sigma) \\
= & \sum_{j=0}^{r-1} \sqrt{2 j+1} \sum_{l=0}^{\infty} \sum_{k=0}^{j}(-1)^{j+k}\left(\begin{array}{c}
j \\
k
\end{array}\right)\left(\begin{array}{c}
j+k \\
k
\end{array}\right) \frac{k !(2 l+1) !}{(2 l+k+2) !} \frac{(-1)^{l} K^{l}}{(2 l+1) !} \tau^{k} \hat{p}_{j}(\sigma) \\
= & \sum_{j=0}^{r-1} \sqrt{2 j+1} \hat{p}_{j}(\sigma) \sum_{l=0}^{\infty} \sum_{k=0}^{j} \frac{(-1)^{j+k+l}(j+k) !}{k !(j-k) !(2 l+k+2) !} \tau^{k} K^{l} .
\end{aligned}
$$

Recall that the generalized hypergeometric function ${ }_{m} F_{n}$ is defined by

$$
{ }_{m} F_{n}\left[\begin{array}{c}
\alpha_{1}, \alpha_{2}, \ldots, \alpha_{m} ; \\
\beta_{1}, \beta_{2}, \ldots, \beta_{n} ; x
\end{array}\right]=\sum_{l=0}^{\infty} \frac{\prod_{i=1}^{m}\left(\alpha_{i}\right)_{l}}{\prod_{i=1}^{n}\left(\beta_{i}\right)_{l}} \frac{x^{l}}{l !},
$$

where $\alpha_{i}$ and $\beta_{i}$ are arbitrary complex numbers, except that $\beta_{i}$ can be neither zero nor a negative integer, and $(z)_{l}$ is the Pochhammer symbol which is defined as

$$
(z)_{0}=1, \quad(z)_{l}=z(z+1) \cdots(z+l-1), \quad l \in \mathbb{N} .
$$

Then, $\mathcal{A}_{\tau, \sigma}$ can be expressed by

$$
\mathcal{A}_{\tau, \sigma}(K)=\sum_{j=0}^{r-1} \sqrt{2 j+1} \hat{p}_{j}(\sigma) \sum_{l=0}^{\infty} \frac{(-1)^{j+l}}{(2 l+2) !}{ }^{2} F_{1}\left[\begin{array}{c}
-j, j+1 ; \\
2 l+3 ;
\end{array}\right] K^{l} .
$$

Likewise, we can obtain

$$
\mathcal{B}_{1, \sigma}(K)=\sum_{j=0}^{r-1} \sqrt{2 j+1} \hat{p}_{j}(\sigma) S_{j}(K)
$$

where $S_{j}(K)$ are given by

$$
\begin{aligned}
S_{2 j}(K) & =(-1)^{j} \frac{(2 j) !}{(4 j+1) !} K^{j}{ }_{0} F_{1}\left[\begin{array}{l}
-; \\
\frac{1}{2} ;
\end{array} ; \frac{K}{16}\right]{ }_{0} F_{1}\left[\begin{array}{c}
-; \\
2 j+\frac{3}{2} ;-\frac{K}{16}
\end{array}\right], \\
S_{2 j+1}(K) & =(-1)^{j} \frac{(2 j+2) !}{(4 j+4) !} K^{j+1}{ }_{0} F_{1}\left[\frac{-}{2} ;-\frac{K}{16}\right]{ }_{0} F_{1}\left[\begin{array}{c}
-; \\
2 j+\frac{5}{2} ;
\end{array}-\frac{K}{16}\right], \quad j=0,1, \ldots
\end{aligned}
$$




\subsection{An example of RKNCr methods}

By letting $K=0$ in the above analysis, we obtain an example of RKNCr methods for solving the general second-order ODEs (20) as

$$
\left\{\begin{array}{l}
q_{d_{i}}=q_{0}+d_{i} h p_{0}-d_{i}^{2} h^{2} \int_{0}^{1} \overline{\mathcal{A}}_{d_{i}, \sigma} \nabla U\left(\sum_{m=1}^{r} q_{d_{m}} l_{m}(\sigma)\right) d \sigma, \quad i=1, \ldots, r, \\
q_{1}=q_{0}+h p_{0}-h^{2} \int_{0}^{1} \overline{\mathcal{A}}_{1, \sigma} \nabla U\left(\sum_{m=1}^{r} q_{d_{m}} l_{m}(\sigma)\right) d \sigma, \\
p_{1}=p_{0}-h \int_{0}^{1} \overline{\mathcal{B}}_{1, \sigma} \nabla U\left(\sum_{m=1}^{r} q_{d_{m}} l_{m}(\sigma)\right) d \sigma,
\end{array}\right.
$$

where $\overline{\mathcal{A}}_{\tau, \sigma}=\sum_{i=0}^{r-1} \int_{0}^{1}(1-\xi) \hat{p}_{i}(\xi \tau) d \xi \hat{p}_{i}(\sigma)$ and $\overline{\mathcal{B}}_{1, \sigma}=\sum_{i=0}^{r-1} \int_{0}^{1} \hat{p}_{i}(\xi) d \xi \hat{p}_{i}(\sigma)$.

Remark 6 It is noted that one can make different choices of $Y_{h}$ and $X_{h}$ and the whole analysis presented in this paper still holds. Different choices will produce different practical methods, and in this paper, we do not go further on this point for brevity.

\section{$9 \quad$ Numerical experiments}

Applying the $r$-point Gauss-Legendre's quadrature to the integral of (12) yields

$$
\left\{\begin{array}{l}
y_{c_{i}}=e^{c_{i} h A} y_{0}+c_{i} h \sum_{j=1}^{r} b_{j} \bar{A}_{c_{i}, c_{j}}(A) g\left(y_{c_{j}}\right), \quad i=1, \ldots, r, \\
y_{1}=e^{h A} y_{0}+h \sum_{j=1}^{r} b_{j} \bar{A}_{1, c_{j}}(A) g\left(y_{c_{j}}\right),
\end{array}\right.
$$

where $c_{j}$ and $b_{j}$ with $j=1, \ldots, r$ are the nodes and weights of the quadrature, respectively. In order to show the efficiency and robustness of our methods, we take $r=2$ and denote the corresponding method by EC2P. Then we choose the same $Y_{h}$ and $X_{h}$ for the functionally fitted energy-preserving method developed in [38, and by this choice, the method becomes the $2 r$ th order RKEPC method given in 23. For this method, we choose $r=2$ and approximate the integral by the Lobatto quadrature of order eight, which is precisely the "extended Labatto IIIA method of order four" in 35. We denote the method as RKEPC2. Another integrator we select for comparisons is the explicit three-stage exponential integrator of order four derived in 34 which is denoted by EEI3s4. It is noted that the first two methods are implicit and we set $10^{-16}$ as the error tolerance and 5 as the maximum number of each fixed-point iteration. It is also remarked that in this paper, we only demonstrate the efficiency of ECr methods when applied to first-order systems, for brevity. Numerical comparisons of TCr and RKNCr methods for solving second-order highly oscillatory systems will be presented elsewhere.

Problem 1. Consider the Duffing equation defined by

$$
\left(\begin{array}{l}
q \\
p
\end{array}\right)^{\prime}=\left(\begin{array}{cc}
0 & 1 \\
-\omega^{2}-k^{2} & 0
\end{array}\right)\left(\begin{array}{l}
q \\
p
\end{array}\right)+\left(\begin{array}{c}
0 \\
2 k^{2} q^{3}
\end{array}\right), \quad\left(\begin{array}{c}
q(0) \\
p(0)
\end{array}\right)=\left(\begin{array}{c}
0 \\
\omega
\end{array}\right) .
$$



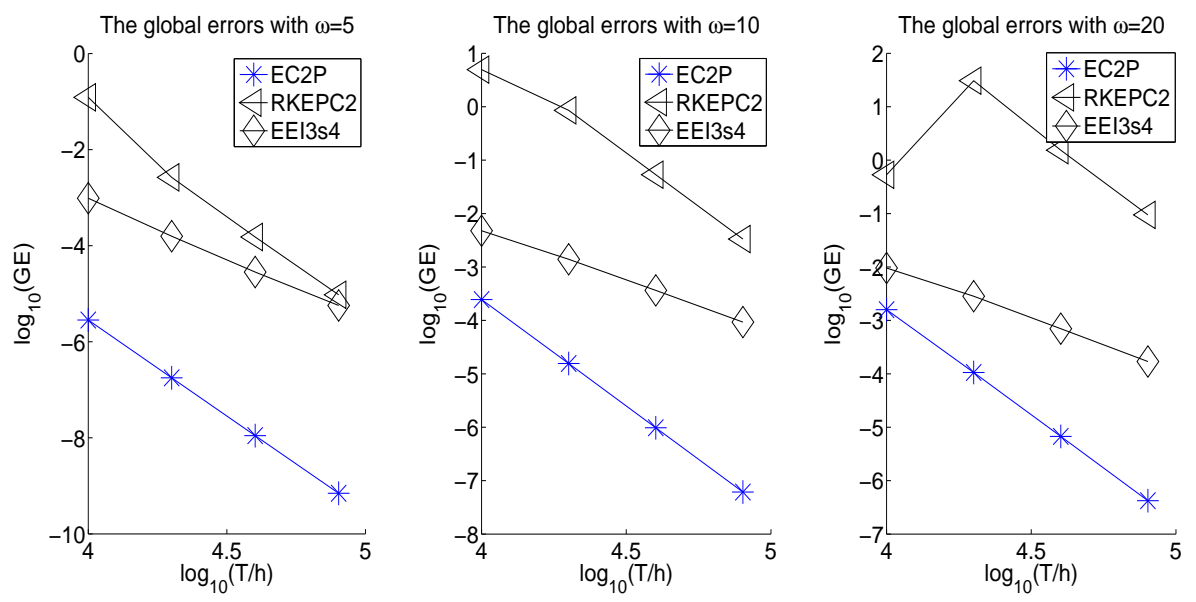

Figure 1: The logarithm of the global error against the logarithm of $T / h$.

It is a Hamiltonian system with the Hamiltonian:

$$
H(p, q)=\frac{1}{2} p^{2}+\frac{1}{2}\left(\omega^{2}+k^{2}\right) q^{2}-\frac{k^{2}}{2} q^{4} .
$$

The exact solution of this system is $q(t)=s n(\omega t ; k / \omega)$ with the Jacobi elliptic function $s n$. Choose $k=0.07, \omega=5,10,20$ and solve the problem in the interval $[0,1000]$ with different stepsizes $h=$ $0.1 / 2^{i}$ for $i=0, \ldots 3$. The global errors are presented in Figure 1. Then, we integrate this problem with the stepsize $h=1 / 100$ in the interval $[0,10000]$. See Figure 2 for the energy conservation for different methods.

Problem 2. Consider the following averaged system in wind-induced oscillation (see [42])

$$
\left(\begin{array}{l}
x_{1} \\
x_{2}
\end{array}\right)^{\prime}=\left(\begin{array}{cc}
-\zeta & -\lambda \\
\lambda & -\zeta
\end{array}\right)\left(\begin{array}{l}
x_{1} \\
x_{2}
\end{array}\right)+\left(\begin{array}{c}
x_{1} x_{2} \\
\frac{1}{2}\left(x_{1}^{2}-x_{2}^{2}\right)
\end{array}\right)
$$

where $\zeta \geq 0$ is a damping factor and $\lambda$ is a detuning parameter. By setting

$$
\zeta=r \cos (\theta), \quad \lambda=r \sin (\theta), \quad r \geq 0, \quad 0 \leq \theta \leq \pi / 2,
$$

this system can be transformed into the scheme (11) with

$$
\begin{aligned}
Q & =\left(\begin{array}{cc}
-\cos (\theta) & -\sin (\theta) \\
\sin (\theta) & -\cos (\theta)
\end{array}\right), \quad M=\left(\begin{array}{cc}
r & 0 \\
0 & r
\end{array}\right) \\
V & =-\frac{1}{2} \sin (\theta)\left(x_{1} x_{2}^{2}-\frac{1}{3} x_{1}^{3}\right)+\frac{1}{2} \cos (\theta)\left(-x_{1}^{2} x_{2}+\frac{1}{3} x_{2}^{3}\right) .
\end{aligned}
$$

Its first integral (conservative case, when $\theta=\pi / 2$ ) or Lyapunov function (dissipative case, when $\theta<\pi / 2)$ is

$$
H=\frac{1}{2} r\left(x_{1}^{2}+x_{2}^{2}\right)-\frac{1}{2} \sin (\theta)\left(x_{1} x_{2}^{2}-\frac{1}{3} x_{1}^{3}\right)+\frac{1}{2} \cos (\theta)\left(-x_{1}^{2} x_{2}+\frac{1}{3} x_{2}^{3}\right) .
$$



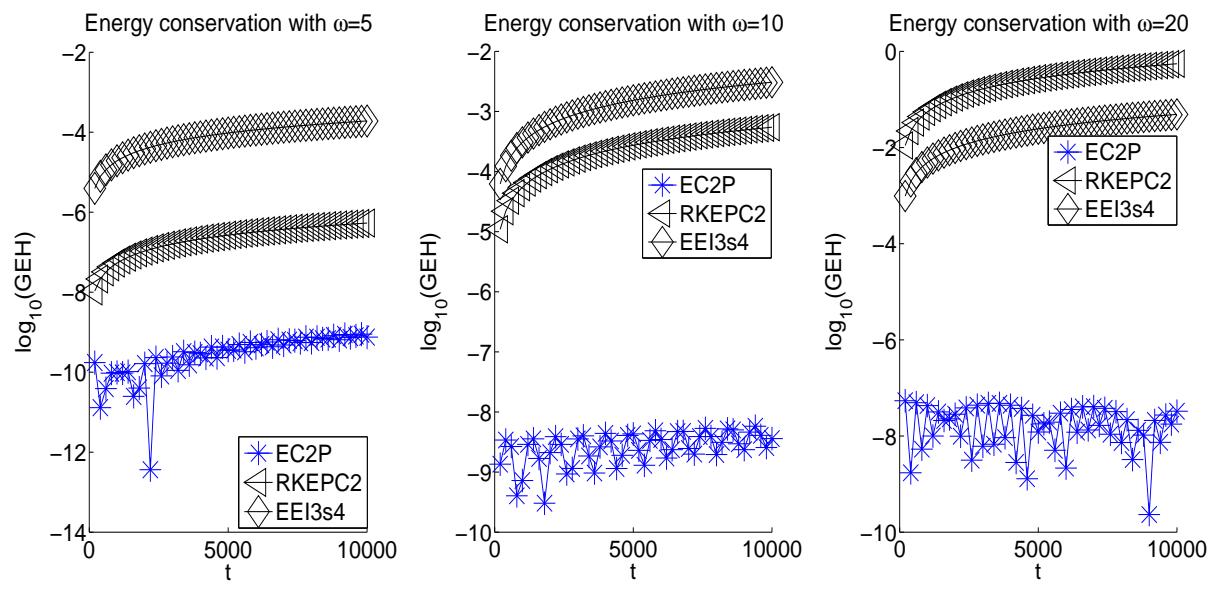

Figure 2: The logarithm of the error of Hamiltonian against $t$.

The initial values are given by $x_{1}(0)=0, x_{2}(0)=1$. Firstly we consider the conservative case and choose $\theta=\pi / 2, r=20$. The problem is integrated in $[0,1000]$ with the stepsize $h=0.1 / 2^{i}$ for $i=1, \ldots, 4$ and the global errors are given in Figure 3 (a). Then we solve this system with the stepsize $h=1 / 200$ in the interval [0,10000] and Figure 3 (b) shows the results of the energy preservation. Secondly we choose $\theta=\pi / 2-10^{-4}$ and this gives a dissipative system. The system is solved in $[0,1000]$ with $h=0.1 / 2^{i}$ for $i=1, \ldots, 4$ and the errors are presented in Figure 4 (a). See Figure 4 (b) for the results of Lyapunov function with $h=1 / 20$. Here we consider the results given by EC2P with a smaller stepsize $h=1 / 1000$ as the 'exact' values of Lyapunov function.

Problem 3. Consider the nonlinear Schrödinger equation (see [16])

$$
i \psi_{t}+\psi_{x x}+2|\psi|^{2} \psi=0, \quad \psi(x, 0)=0.5+0.025 \cos (\mu x)
$$

with the periodic boundary condition $\psi(0, t)=\psi(L, t)$. Following [16], we choose $L=4 \sqrt{2} \pi$ and $\mu=2 \pi / L$. The initial condition chosen here is in the vicinity of the homoclinic orbit. Using $\psi=p+\mathrm{i} q$, this equation can be rewritten as a pair of real-valued equations

$$
\begin{aligned}
& p_{t}+q_{x x}+2\left(p^{2}+q^{2}\right) q=0 \\
& q_{t}-p_{x x}-2\left(p^{2}+q^{2}\right) p=0
\end{aligned}
$$

Discretising the spatial derivative $\partial_{x x}$ by the pseudospectral method given in [16], this problem is converted into the following system:

$$
\left(\begin{array}{l}
\mathbf{p} \\
\mathbf{q}
\end{array}\right)^{\prime}=\left(\begin{array}{cc}
0 & -D_{2} \\
D_{2} & 0
\end{array}\right)\left(\begin{array}{l}
\mathbf{p} \\
\mathbf{q}
\end{array}\right)+\left(\begin{array}{c}
-2\left(\mathbf{p}^{2}+\mathbf{q}^{2}\right) \cdot \mathbf{q} \\
2\left(\mathbf{p}^{2}+\mathbf{q}^{2}\right) \cdot \mathbf{p}
\end{array}\right)
$$

where $\mathbf{p}=\left(p_{0}, p_{1}, \ldots, p_{N-1}\right)^{\top}, \mathbf{q}=\left(q_{0}, q_{1}, \ldots, q_{N-1}\right)^{\top}$ and $D_{2}=\left(D_{2}\right)_{0 \leq j, k \leq N-1}$ is the pseudospec- 

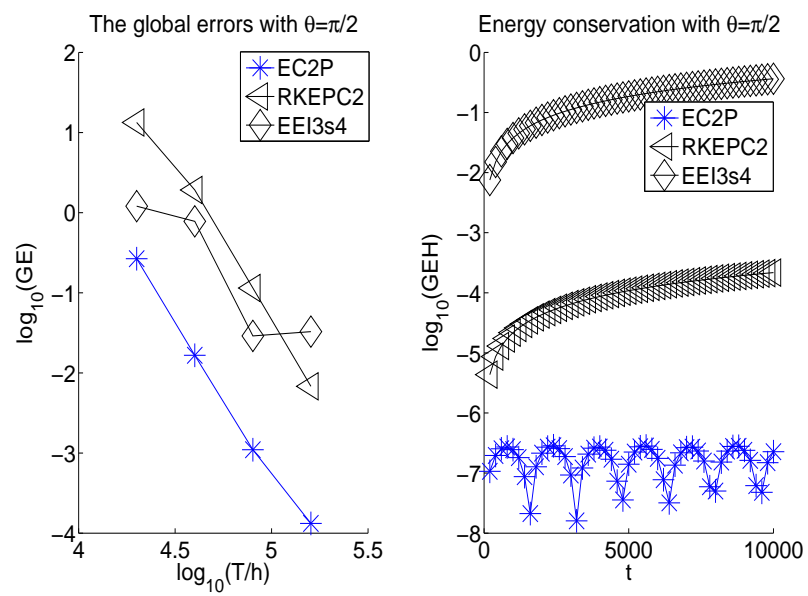

Figure 3: (a) The logarithm of the global error against the logarithm of $T / h$. (b) The logarithm of the error of Hamiltonian against $t$.
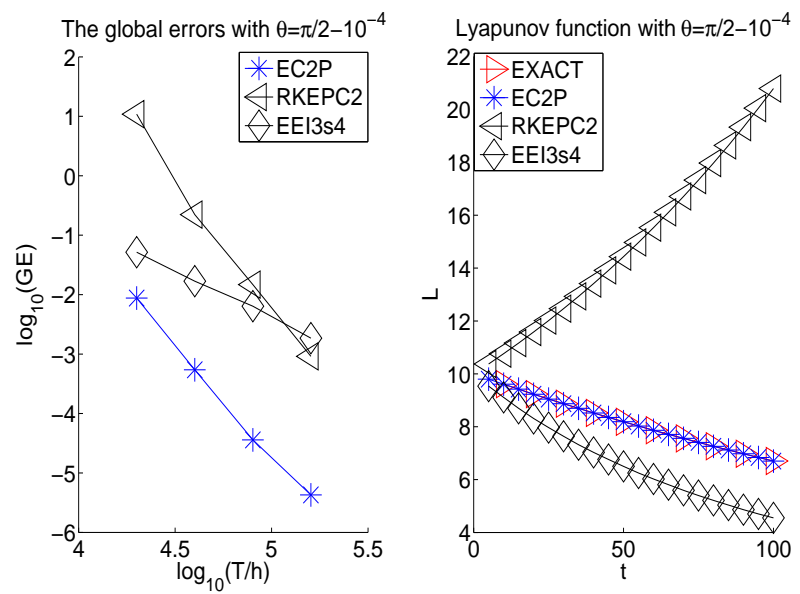

Figure 4: (a) The logarithm of the global error against the logarithm of $T / h$. (b) The results of the Lyapunov function against $t$. 

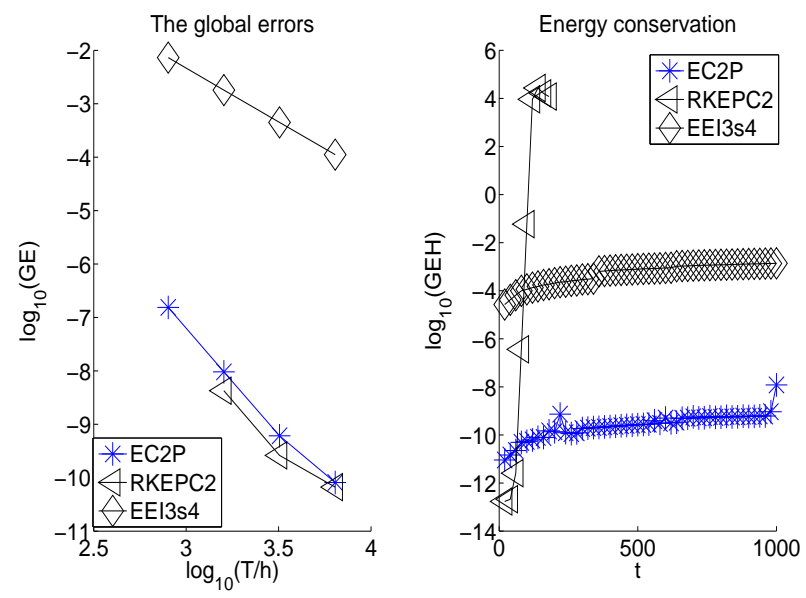

Figure 5: (a) The logarithm of the global error against the logarithm of $T / h$. (b) The logarithm of the error of Hamiltonian against $t$.

tral differential matrix defined by:

$$
\left(D_{2}\right)_{j k}= \begin{cases}\frac{1}{2} \mu^{2}(-1)^{j+k+1} \frac{1}{\sin ^{2}\left(\mu\left(x_{j}-x_{k}\right) / 2\right)}, & j \neq k, \\ -\mu^{2} \frac{2(N / 2)^{2}+1}{6}, & j=k,\end{cases}
$$

with $x_{j}=j \frac{L}{N}$ for $j=0,1, \ldots, N-1$. The Hamiltonian of (41) is

$$
H(\mathbf{p}, \mathbf{q})=\frac{1}{2} \mathbf{p}^{\boldsymbol{\top}} D_{2} \mathbf{p}+\frac{1}{2} \mathbf{q}^{\boldsymbol{\top}} D_{2} \mathbf{q}+\frac{1}{2} \sum_{i=0}^{N-1}\left(p_{i}^{2}+q_{i}^{2}\right)^{2} .
$$

We choose $N=128$ and first solve the problem in the interval $[0,10]$ with $h=0.1 / 2^{i}$ for $i=3, \ldots, 6$.

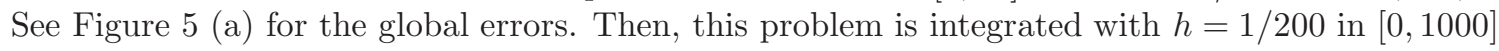
and the energy conservation is presented in Figure 5(b).

It can be concluded from these numerical experiments that our EC2P method definitely shows higher accuracy, more excellent invariant-preserving property, and prominent long-term behavior in the numerical simulations, than the other effective methods in the literature.

\section{Concluding remarks and discussions}

Exponential integrators have constituted an important class of methods for the numerical simulation of first-order ODEs, including the semi-discrete nonlinear Schrödinger equation etc. Finite element methods for ODEs can date back to early 1960s and they have been investigated by many researchers. In this paper, combining the ideas of these two effective methods, we derived and analysed a new kind of exponential collocation methods for the conservative or dissipative system (11). We have also rigorously analysed the properties including existence and uniqueness, and algebraic 
order. It has been proved that our novel methods can be arbitrary-order accuracy as well as exactly or nearly preserving first integrals or Lyapunov functions. The application of our methods in stiff gradient systems was discussed. The efficiency and superiority of the new methods were numerically demonstrated by performing some experiments. By the analysis of this paper, arbitrary-order energy-preserving methods were presented for second-order highly oscillatory/general systems.

Last but not least, it is noted that there are still some issues of the methods which can be further considered.

- The error bounds and convergence properties of the methods will be discussed in another work.

- Another issue for exploration is the application of our methodology in PDEs such as nonlinear Schördinger equations and wave equations. We have derived exponential integrators to preserve the continuous energy of Schördinger equations (see [53]).

- The application of our methodology in other ODEs such as general gradient systems and Poisson systems will also be considered.

\section{Acknowledgement}

The authors are grateful to Professor Christian Lubich for his careful reading of the manuscript and for his helpful comments. It is also his idea that motivates Section 7 of this manuscript.

\section{References}

[1] A. H. Al-Mohy and N. J. Higham, Computing the action of the matrix exponential, with an application to exponential integrators, SIAM J. Sci. Comput., 33 (2011), pp. 488-511.

[2] J. Barrett and J. Blowey, Finite element approximation of an Allen-Cahn/Cahn-Hilliard system, IMA J. Numer. Anal., 22 (2002), pp. 11-71.

[3] H. Berland, B. Owren, and B. Skaflestad, B-series and order conditions for exponential integrators, SIAM J. Numer. Anal., 43 (2005), pp. 1715-1727.

[4] P. Betsch and P. Steinmann, Inherently energy conserving time finite elements for classical mechanics, J. Comput. Phys., 160 (2000), pp. 88-116.

[5] P. Betsch and P. Steinmann, Conservation properties of a time FE method, I. Time-stepping schemes for N-body problems, Int. J. Numer. Meth. Eng., 49 (2000), pp. 599-638.

[6] L. Brugnano, F. Iavernaro, and D. Trigiante, Hamiltonan Boundary Value Methods (Energy Preserving Discrete Line Integral Methods), J. Numer. Anal. Ind. Appl. Math., 5 (2010), pp. 13-17.

[7] L. Brugnano, F. Iavernaro, and D. Trigiante, Energy- and quadratic invariants-preserving integrators based upon Gauss-Collocation formulae, SIAM J. Numer. Anal., 50 (2012), pp. 28972916.

[8] J. C. Butcher, Trees, B-series and exponential integrators, IMA J. Numer. Anal., 30 (2009), pp. 131-140. 
[9] M. Caliari and A. Ostermann, Implementation of exponential Rosenbrock-type integrators, Appl. Numer. Math., 59 (2009), pp. 568-581.

[10] M. Calvo, M. P. Laburta, J. I. Montijano, and L. Rández, Projection methods preserving Lyapunov functions, BIT, 50 (2010), pp. 223-241.

[11] M. Calvo and C. Palencia, A class of explicit multistep exponential integrators for semilinear problems, Numer. Math., 102 (2006), pp. 367-381.

[12] B. Cano, A Gonzalez-Pachon, Projected explicit Lawson methods for the integration of Schrödinger equation, Num. meth. for PDEs, 2013

[13] E. Celledoni, D. Cohen, and B. Owren, Symmetric exponential integrators with an application to the cubic Schrödinger equation, Found. Comput. Math., 8 (2008), pp. 303-317.

[14] E. Celledoni, R. I. Mclachlan, B. Owren, and G. R. W. Quispel, Energy-preserving integrators and the structure of B-series, Found. Comput. Math., 10 (2010), pp. 673-693.

[15] E. Celledoni, B. Owren, and Y. Sun, The minimal stage, energy preserving Runge-Kutta method for polynomial Hamiltonian systems is the averaged vector field method, Math. Comp., 83 (2014), pp. 1689-1700.

[16] J. B. Chen and M. Z. Qin, Multisymplectic Fourier pseudospectral method for the nonlinear Schrödinger equation, Electron. Trans. Numer. Anal., 12 (2001), pp. 193-204.

[17] D. Cohen and E. Hairer, Linear energy-preserving integrators for Poisson systems, BIT, 51 (2011), pp. 91-101.

[18] D. Cohen, E. Hairer, and C. Lubich, Numerical energy conservation for multi-frequency oscillatory differential equations, BIT, 45 (2005), pp. 287-305.

[19] L. Einkemmer, M. Tokman, and J. Loffeld, On the performance of exponential integrators for problems in magnetohydrodynamics, J. Comput. Phys., 330 (2017), pp. 550-565.

[20] J. M. Franco, New methods for oscillatory systems based on ARKN methods, Appl. Numer. Math., 56 (2006), pp. 1040-1053.

[21] B. García-Archilla, J. M. Sanz-Serna, and R. D. Skeel, Long-time-step methods for oscillatory differential equations, SIAM J. Sci. Comput., 20 (1999), pp. 930-963.

[22] V. Grimm and M. Hochbruck, Error analysis of exponential integrators for oscillatory secondorder differential equations, J. Phys. A: Math. Gen., 39 (2006), pp. 5495-5507.

[23] E. Hairer, Energy-preserving variant of collocation methods, J. Numer. Anal. Ind. Appl. Math., 5 (2010), pp. 73-84.

[24] E. Hairer and C. Lubich, Energy-diminishing integration of gradient systems, IMA J. Numer. Anal., 34 (2014), pp. 452-461.

[25] E. Hairer, C. Lubich, and G. Wanner, Geometric Numerical Integration: Structure-Preserving Algorithms for Ordinary Differential Equations, 2nd edn. Springer-Verlag, Berlin, Heidelberg, 2006. 
[26] E. Hairer and G. Wanner, Solving Ordinary Differential Equations II. Stiff and DifferentialAlgebraic Problems, Springer Series in Computational Mathematics 14, 2nd edn. SpringerVerlag, Berlin, Heidelberg, 1996.

[27] P. Hansbo, A note on energy conservation for Hamiltonian systems using continuous time finite elements, Commu. Numer. Meth. Engi., 17 (2001), pp. 863-869

[28] M. Hochbruck and C. Lubich, On Krylov subspace approximations to the matrix exponential operator, SIAM J. Numer. Anal., 34 (1997), pp. 1911-1925.

[29] M. Hochbruck and C. Lubich, A Gautschi-type method for oscillatory second-order differential equations, Numer. Math., 83 (1999), pp. 403-426.

[30] M. Hochbruck, C. Lubich, and H. Selhofer, Exponential integrators for large systems of differential equations, SIAM J. Sci. Comput., 19 (1998), pp. 1552-1574.

[31] M. Hochbruck and A. Ostermann, Exponential Runge-Kutta methods for parabolic problems, Appl. Numer. Math., 53 (2005), pp. 323-339.

[32] M. Hochbruck and A. Ostermann, Explicit exponential Runge-Kutta methods for semilineal parabolic problems, SIAM J. Numer. Anal., 43 (2005), pp. 1069-1090.

[33] M. Hochbruck and A. Ostermann, Exponential integrators, Acta Numer., 19 (2010), pp. 209286.

[34] M. Hochbruck, A. Ostermann, and J. Schweitzer, Exponential rosenbrock-type methods, SIAM J. Numer. Anal., 47 (2009), pp. 786-803.

[35] F. Iavernaro and D. Trigiante, High-order symmetric schemes for the energy conservation of polynomial Hamiltonian problems, J. Numer. Anal. Ind. Appl. Math., 4 (2009), pp. 87-101.

[36] A. Iserles, Think globally, act locally: solving highly-oscillatory ordinary differential equations, Appl. Numer. Math., 43 (2002), pp. 145- 160.

[37] Y.W. Li and X. Wu, Exponential integrators preserving first integrals or Lyapunov functions for conservative or dissipative systems, SIAM J. Sci. Comput., 38 (2016), pp. 1876-1895.

[38] Y.W. Li and X. Wu, Functionally fitted energy-preserving methods for solving oscillatory nonlinear Hamiltonian systems, SIAM J. Numer. Anal., 54 (2016), pp. 2036-2059.

[39] C. Lubich and A. Ostermann, Runge-Kutta methods for parabolic equations and convolution quadrature, Math. Comp., 60 (1993), pp. 105-131.

[40] C. Lubich and A. Ostermann, Runge-Kutta time discretization of reaction-diffusion and NavierStokes equations: nonsmooth-data error estimates and applications to long-time behaviour, Appl. Numer. Math., 22 (1996), pp. 279-292.

[41] R. I. McLachlan and G. R. W. Quispel, Discrete gradient methods have an energy conservation law, Disc. Contin. Dyn. Syst., 34 (2014), pp. 1099-1104.

[42] R. I. Mclachlan, G. R. W. Quispel, and N. Robidoux, A unified approach to Hamiltonian systems, Poisson systems, gradient systems, and systems with Lyapunov functions or first integrals, Phys. Rev. Lett., 81 (1998), pp. 2399-2411 
[43] R. I. McLachlan, G. R. W. Quispel, and N. Robidoux, Geometric integration using discrete gradient, Philos. Trans. R. Soc. Lond. A, 357 (1999), pp. 1021-1045.

[44] L. Mei and X. Wu, Symplectic exponential Runge-Kutta methods for solving nonlinear Hamiltonian systems, J. Comput. Phys., 338 (2017), pp. 567-584.

[45] Y. Miyatake, An energy-preserving exponentially-fitted continuous stage Runge-Kutta method for Hamiltonian systems, BIT, 54 (2014), pp. 777-799.

[46] Y. Miyatake, A derivation of energy-preserving exponentially-fitted integrators for Poisson systems, Comput. Phys. Comm., 187 (2015), pp. 156-161.

[47] A. Ostermann, M. Thalhammer, and W. M. Wright, A class of explicit exponential general linear methods, BIT, 46 (2006), pp. 409-431.

[48] G. R. W. Quispel and D. I. McLaren, A new class of energy-preserving numerical integration methods, J. Phys. A, 41 (045206) (2008), 7pp.

[49] W. Tang and Y. Sun, Time finite element methods: A unified framework for the numerical discretizations of ODEs, Appl. Math. Comput., 219 (2012), pp. 2158-2179.

[50] B. Wang, A. Iserles, and X. Wu, Arbitrary-order trigonometric Fourier collocation methods for multi-frequency oscillatory systems, Found. Comput. Math., 16 (2016), pp. 151-181.

[51] B. Wang, F. Meng, and Y. Fang, Efficient implementation of RKN-type Fourier collocation methods for second-order differential equations, Appl. Numer. Math., 119 (2017), pp. 164-178.

[52] B. Wang, X. Wu, The formulation and analysis of energy-preserving schemes for solving high-dimensional nonlinear Klein-Gordon equations, IMA J. Numer. Anal., in press, doi:10.1093/imanum/dry047.

[53] B. Wang and $\mathrm{X}$. Wu, Exponential collocation methods for efficiently solving the cubic Schrödinger equation, arXiv:1802.02831v2

[54] B. Wang and X. Wu, A new high precision energy-preserving integrator for system of oscillatory second-order differential equations, Phys. Lett. A, 376 (2012), pp. 1185-1190.

[55] B. Wang, X. Wu, and F. Meng, Trigonometric collocation methods based on Lagrange basis polynomials for multi-frequency oscillatory second-order differential equations, J. Comput. Appl. Math., 313 (2017), pp. 185-201.

[56] B. Wang, X. Wu, F. Meng, and Y. Fang, Exponential Fourier collocation methods for solving first-order differential equations, J. Comput. Math., 35 (2017), pp. 711-736.

[57] B. Wang, H. Yang, and F. Meng, Sixth order symplectic and symmetric explicit ERKN schemes for solving multi-frequency oscillatory nonlinear Hamiltonian equations, Calcolo, 54 (2017), pp. 117-140.

[58] X. Wu, B. Wang, Recent Developments in Structure-Preserving Algorithms for Oscillatory Differential Equations, Springer Nature Singapore Pte Ltd, 2018. 
[59] X. Wu, B. Wang, and W. Shi, Efficient energy preserving integrators for oscillatory Hamiltonian systems, J. Comput. Phys., 235 (2013), pp. 587-605.

[60] X. Wu, B. Wang, and J. Xia, Explicit symplectic multidimensional exponential fitting modified Runge-Kutta-Nyström methods, BIT, 52 (2012), pp. 773-795.

[61] X. Wu, X. You, W. Shi, and B. Wang, ERKN integrators for systems of oscillatory second-order differential equations, Comput. Phys. Comm., 181 (2010), pp. 1873-1887.

[62] X. Wu, X. You, and B. Wang, Structure-preserving algorithms for oscillatory differential equations, Springer-Verlag, Berlin, Heidelberg, 2013. 